\title{
A simulation study on the relative role of age groups under differing pertussis transmission scenarios
}

Ana I. Bento ${ }^{1,2 *}$, Aaron A. King ${ }^{3,4}$, Pejman Rohani ${ }^{1,2,5}$

1 Odum School of Ecology, University of Georgia, Athens, GA, USA

2 Center for the Ecology of Infectious Diseases, University of Georgia, Athens, GA, USA

3 Center for the Study of Complex Systems, University of Michigan, Ann Arbor, MI, USA

4 Department of Ecology \& Evolutionary Biology, University of Michigan, Ann Arbor, MI, USA

5 Department of Infectious Diseases, University of Georgia, Athens, GA, USA

* E-mail: anabento@uga.edu

\section{Abstract}

Pertussis has resurged in many countries where it was once regarded as under control, with the recent outbreaks showing a shift in incidence towards teens and older individuals. Here, using an age-stratified transmission model, we tested two potential causes for underlying changes in pertussis transmission dynamics. We did so assuming hypothesized mechanisms supporting present-day pertussis epidemiology: (I) improved diagnostics, (II) acellular vaccines leading to asymptomatic transmission (III) both. We used the relative risks and odds ratio methods to examine the impact of these differing assumptions on signatures of relative roles of key age groups through time, allowing us to explore those age cohorts that disproportionately account for transmission. Our findings show that for epidemics after the vaccine switch, a scenario with increased adult reporting and no asymptomatic transmission reflect a loss of signal, where no age group appears to be key. While scenarios with asymptomatic transmission, reflect a population where children (1-10 years old) are still disproportionally at risk. These results demonstrate that understanding the underlying transmission mechanisms in a population are paramount for vaccination policies in attaining herd immunity and eventually eradication.

Keywords: Pertussis, whooping cough, relative risks transmission, asymptomatic infection, disease dynamical modeling, vaccination

\section{Introduction}

Pertussis is a highly transmissible vaccine-preventable respiratory disease $[1,2,3,4]$. With the implementation of routine immunization in the 1950 s with whole-cell pertussis (DTP) vaccines, pertussis 
incidence decreased considerably [5, 6, 7] allowing this disease to become a candidate for eradication. However, recently, countries boasting high pertussis vaccine coverage, such as the UK and the US, have experienced increased incidence accompanied with infant pertussis-linked deaths $[4,7,8,9,10]$.

Historically considered a childhood disease, these resurgence events are characterized by a remarkable shift in the age-distribution of cases $[11,2,4,9,10]$. This is reflected by the increasing incidence in adolescents and adults that may currently be contributing disproportionately to transmission, perhaps due to higher contact rates or lower immunity $[12,13,14,15,10]$. Thus, subsequently being overrepresented in incidence reports as an epidemic takes off.

Potential explanations for these patterns include improved diagnostic testing, leading to better surveillance and higher reporting $[8,16,17]$, and the switch from the DTP to acellular pertussis vaccines $(\mathrm{DTaP})[18,19,20]$. Reporting rates for pertussis cases vary by age group, thus the leading to ambiguity of age-related transmission roles [21]. There is a growing concern that the DTaP vaccine might be an insufficient control measure [17]. In animal models, it has been suggested that acellular vaccines may not prevent infection and, additionally, allow for asymptomatic transmission [22]. This pattern of resurgence could also be consistent with the long-term dynamical consequences of lower vaccination coverage, resulting in a build-up of susceptible adults who were neither vaccinated nor infected [8].

The uncertainty surrounding the role of different age cohorts further complicates matters. We investigate potential changes in age-specific drivers of pertussis transmission by performing a simulation study, based on an age-structured model of pertussis transmission that incorporates England and Wales demography and contact patterns, historical immunization practices, the introduction of serological and PCR diagnostic methods in 2001 and the switch to the acellular vaccine (DTaP) in 2004.

Focusing on two different epidemics - before and after changes in vaccine and diagnostics - we evaluate scenarios of asymptomatic transmission and increased adult reporting. For the different scenarios, we attempt to characterize the role of different age groups by comparing the patterns of incidence before and after an epidemic peak. For the different scenarios, we use relative risk and odds ratio analyses - which calculate the ratio of the probability of an event occurring in one group to the probability of it occurring in another group [23]. We thus try to identify age groups at higher risk, suggestive of enhanced susceptibility to pertussis infection and/or higher contact rates [21]. Measuring age cohorts" risks in the different scenarios, will further allows us to understand the knock-on consequences at the population level of changes in both vaccine and diagnostics.

Contrasting these different scenarios and odds ratio measures with actual incidence reports, for a given country, we may be able to better understand recent epidemiological data and the underlying 
transmission dynamics. Thus, detecting possible age cohorts driving transmission could be crucial for an adaptive assessment of control policies.

\section{Methods}

\section{Model formulation}

\section{A baseline model of pertussis transmission}

We implemented an age-structured, compartmental model of pertussis transmission, building on previously proposed models that have carried out statistical fitting of transmission models to pertussis incidence reports $[24,25,26,27]$. The model is an extension of the classic SIR model with routine vaccination, implemented as a system of ordinary differential equations. It incorporates empirical agespecific contact rates from the POLYMOD study in Great Britain [28].

Our model is comprised of 18 age classes: 12 1-month infant age classes and 1-4 years, 5-9 years, 10-14 years, 15-19 years, 20-44 years, $45-$ years. Individuals are born susceptible (at a rate $\mu$ ). Susceptible individuals become exposed through contact with infectious individuals and become infectious. After recovering, individuals are immune to further infection. We assume infection-derived immunity to be practically life lifelong, based on previous epidemiological studies of pertussis in England and Wales [24], Sweden [25] and Thailand [26], which found that repeat infections contributed very little to transmission [29]. Individuals in the routine vaccinated class (V) may lose immunity at rate $\epsilon$. This rate of waning is exponentially distributed, meaning that many will lose immunity faster than the population average. We assume routine vaccination to also afford protection lasting, on average, 75 years, while being aware that there is considerable discussion regarding the nature and duration of pertussis vaccine protection $[30,31,20]$. The average infectious period $(1 / \gamma)$ was fixed at 21 days [8, 32]. The per capita birth and death rates were fixed at $\mu=1 / 75$ per year. Individuals who are infected, have a coarse age-specific reporting probability $\iota$ of their infection status being recorded (Table 1 ). We assumed a basic reproduction number, $R_{0}$ of 10 [20]. The model equations and associated parameter values are presented in detail in the Supplementary materials and in Table 1.

At time $t_{a}$ we introduce vaccination. Individuals may be vaccinated with either whole cell (before time $t_{b}$ ) or acellular vaccine (after time $t_{b}$ ). The model explicitly models acellular vaccine replacing whole cell vaccine. Routine infant vaccination occurs at 2, 4, and 6 months of age in order to mimic the protective effects afforded following the receipt of three doses of pertussis vaccine. It is implemented by 
moving, with probability $\rho$ (corresponding to the vaccine coverage), those susceptible individuals who age out of their 2-, 4-, or 6-month age categories into the vaccinated (V) class.

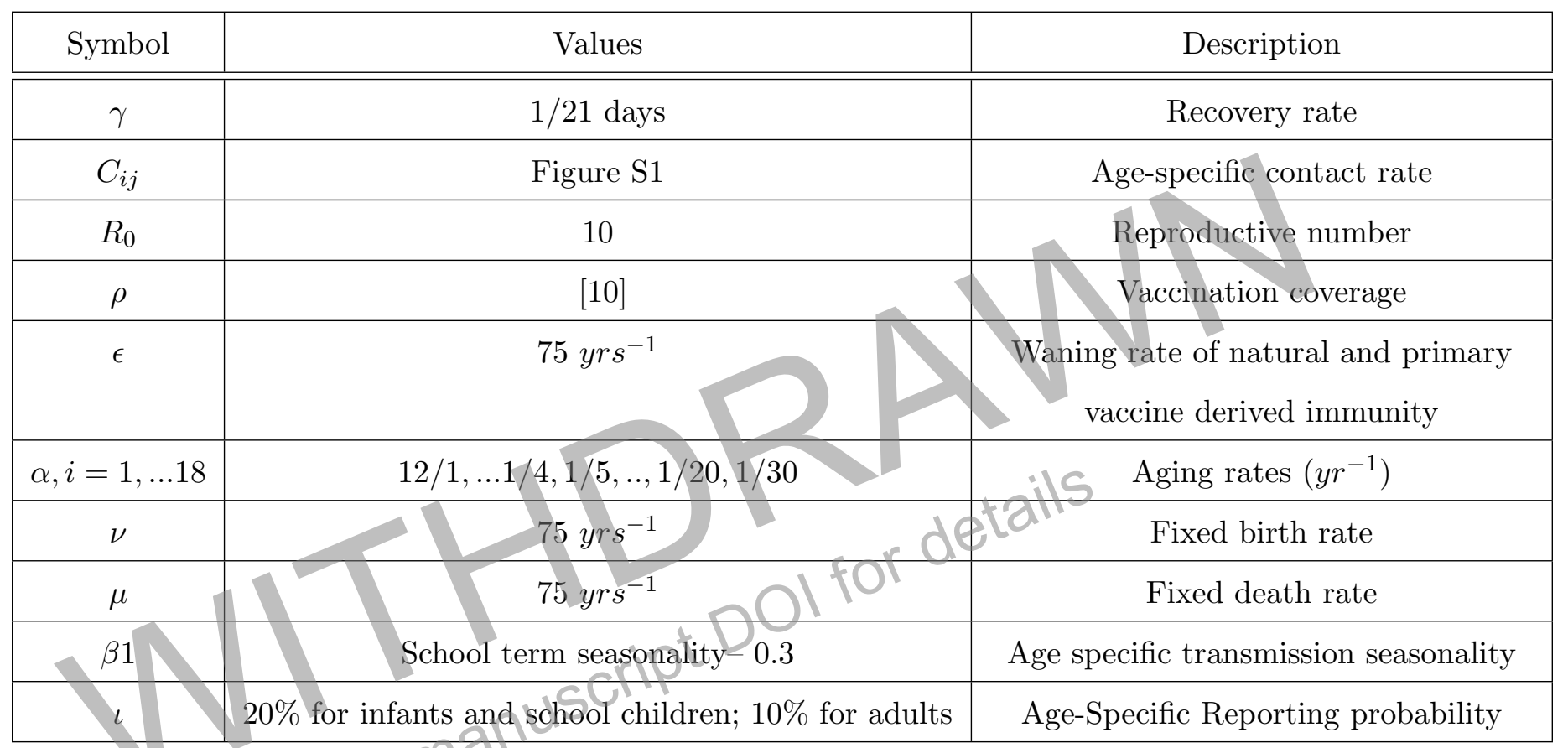

Table 1: Fixed model parameters for pertussis-like transmission simulation study.

\section{Contact network data and $R_{0}$ estimation}

Our model incorporated empirical age-specific contact rates from the POLYMOD study in Great Britain [28], corrected for reciprocity as detailed Riolo et al. [27]. We constructed a WAIFW (Who Acquires Infection From Whom) matrix, to describe the transmission rate between different age groups (Figure S1). The Basic Reproduction Number, $R_{0}$, for our model was calculated using the Next Generation Method [33]. See supplementary materials for details.

\section{Model scenarios}

1. Scenario I - Baseline model with no changes in reporting and a change in vaccine from whole cell to acellular.

2. Scenario II - Asymptomatic carriage after time $t_{b}$, to simulate a vaccination switch from DTP to DTaP. An added compartment to the baseline model: Infected Asymptomatic (A). Individuals who are vaccinated with Tdap may become infectious but will not show symptoms. 
3. Scenario III - Increased adult reporting after $t_{c}$, to simulate introduction of PCR testing (from $10 \%$ to $20 \%)$.

4. Scenario IV - Both asymptomatic carriage after time $t_{b}$ and increased adult reporting after $t_{c}$.

\section{Reporting probability}

From the simulated data at each time $t$, for both scenarios - with and without asymptomatic transmission - we sampled the true infections $I_{t}$ to mimic under reporting, assuming sampling is negative-binomially distributed.

$$
C_{t} \backsim \operatorname{Negbin}\left(\text { mean }=\iota I_{t}, \text { size }=\frac{1}{\theta}\right)
$$

where $C_{t}$ are the case reports, $\iota$ changes depending on the age class, and also in a scenario with changes in reporting in the older age classes at specific year in time ( $\iota$ from $10 \%$ to $20 \%$ ).

\section{Asymptomatic carriage}

Individuals vaccinated with DTaP move into a vaccinated class where they can become asymptomatically infected. We assume no difference in transmissibility between symptomatic and asymptomatic individuals in scenarios II and IV. In scenarios V and VI, asymptomatic individuals are $\frac{1}{2}$ as infectious than non vaccinated infected individuals, but the infectious period is doubled.

\section{Age specific relative risks during an outbreak}

We attempted to characterize the role of different age groups by comparing the patterns of incidence before and after the epidemic peak [21]. We do this for all scenarios.

\section{Defining periods}

We defined the peak week as the calendar week in two specific outbreak years: one in the period before implementation of increased reporting in adults and before switching vaccination types (associated with asymptomatic carriage). Because the data are noisy and do not have a consistently timed annual peak, we characterized the pre- and post-peak periods (time windows) by smoothing the data using a cubic spline with 3 knots. The pre-peak is "take off' period before the peak of the epidemic. The post-peak is the "tail" of the epidemic, it was determined by the same method using the cut off week with the emergence of a smaller peak in incidence. This choice of spline for determining pre and post windows 
was validated by a generalized additive model (GAM) [34] and by segmented regression [35] (Refer to supplementary materials for details and figure S5).

\section{Relative risks calculation}

Additionally we estimated the relative role that different age groups play in the transmission of pertussis infection, as previously used by Worby and colleagues [21]. The population was split into groups. We estimate the relative risk for each age group defined as:

$$
R R(i)=\frac{B(i)}{A(i)}
$$

Where $\mathrm{B}(\mathrm{i})$ is the proportion of the cases in age group $i$ over all the cases in the pre-peak period and $A(i)$ is the proportion for the cases in that same age group $i$ overall cases during the post-peak period.

In the pre- and post-peak periods, we calculate the fraction (ratio) of cases which occur in each age group. We estimate the relative risk for each age group $i$ as ratio of the fraction of pre-peak cases in age group $i$ to the fraction of post-peak cases in age group $i$. This depicts a probability of a specific group being at high risk before the epidemic takes off when compared to post peak period. Thus comparing the periods before and after the epidemic's peak. This allows us to account for difference in overall incidence between age groups and between pre- and post-peak periods. The $95 \%$ confidence intervals were calculated for relative risk following Lachin [23], where $\ln (R R(i))$ is approximately normal.

\section{Odds ratio calculation}

In order to compare the extent to which different age groups are overrepresented in the pre-peak period, we can look at the odds-ratio between pairs of these relative risks of two different age groups. This gives us a relative measure of effect, allowing us to compare between age groups.

We estimate the odds ratio to characterize which age group when compared with another age group shows a more pronounced susceptibles' depletion during the period approaching the outbreak - pre-peak period:

$$
O R(i, j)=\frac{R R(i)}{R R(j)}
$$


bioRxiv preprint doi: https://doi.org/10.1101/247007; this version posted January 11,2018 . The copyright holder for this preprint (which was not certified by peer review) is the author/funder. All rights reserved. No reuse allowed without permission.

Relative role of age groups

where $i$ and $j$ are age groups as define for the previous methods. The $95 \%$ confidence intervals were calculated for odds ratios following Lachin [23], where $\ln (O R(i, j))$ is approximately normal.

\section{Results}

\section{Baseline Scenario - No changes in adult reporting, no asymptomatic transmission}

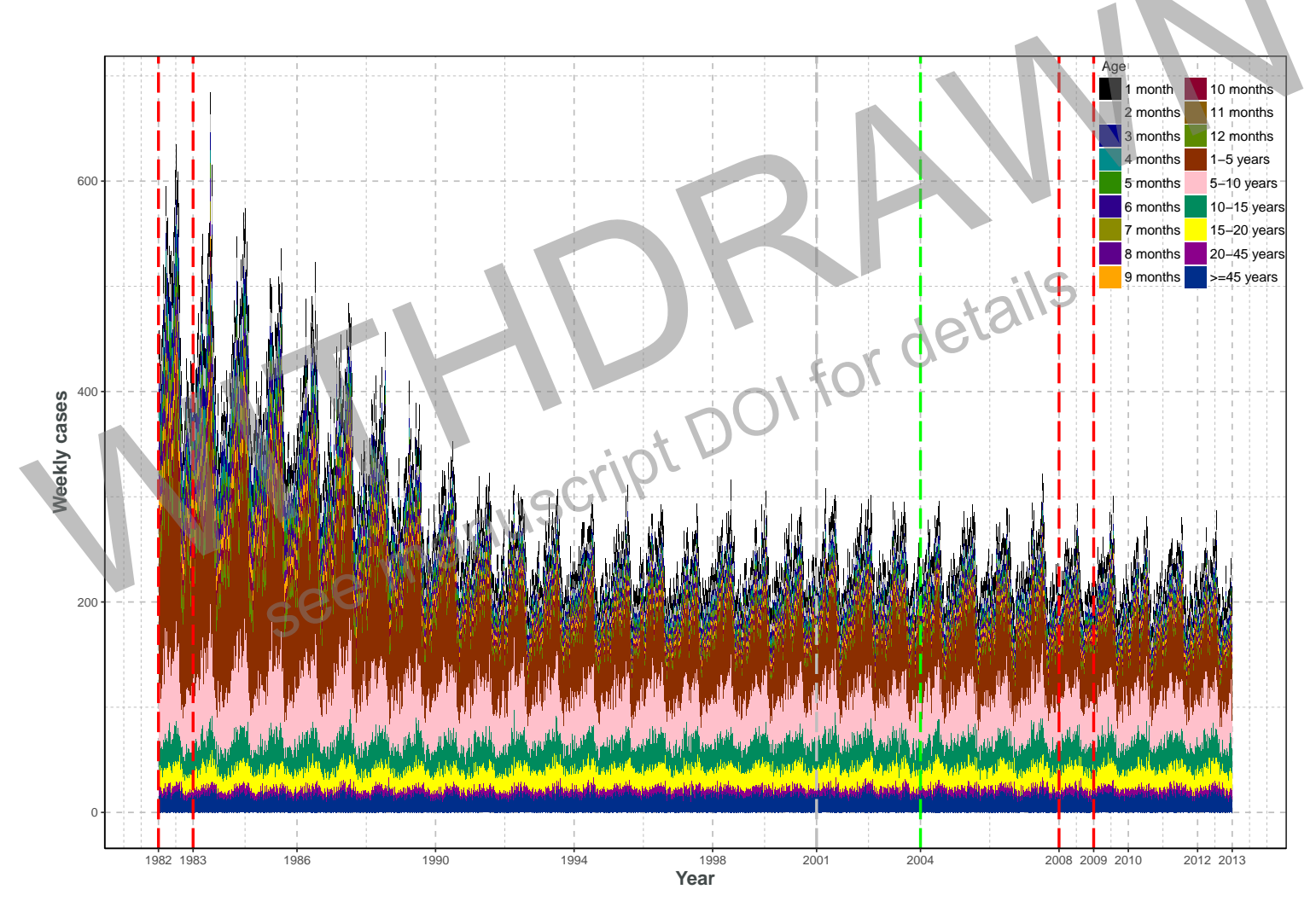

Figure 1: Simulated time series: Baseline scenario - No changes in adult reporting, no asymptomatic transmission. Colors in the time series represent pertussis weekly incidence for each age class. Red vertical dashed lines encompass the epidemic years (1982 and 2008) used for the odds ratio calculations. Grey vertical dashed line shows year (2001) of changes in reporting (baseline scenario does not simulate increase in adult reporting). Green vertical dashed line represents the year DTP was replaced by DaTP (2004).

The simulated time series show noo changes in either vaccination or adult reporting. 


\section{Odds ratios}

\section{Baseline scenario: Peak week}

Two outbreaks in detail are show here, before and after vaccine swith from DTP to DTaP. In both periods peak week is at week 28 , with the epoch before vaccine switch showing higher incidence fore younger age classes. The ascending period and descending windows of the after vaccine switch are a few weeks larger, as calculated using the GAM methodology (Figure S5).
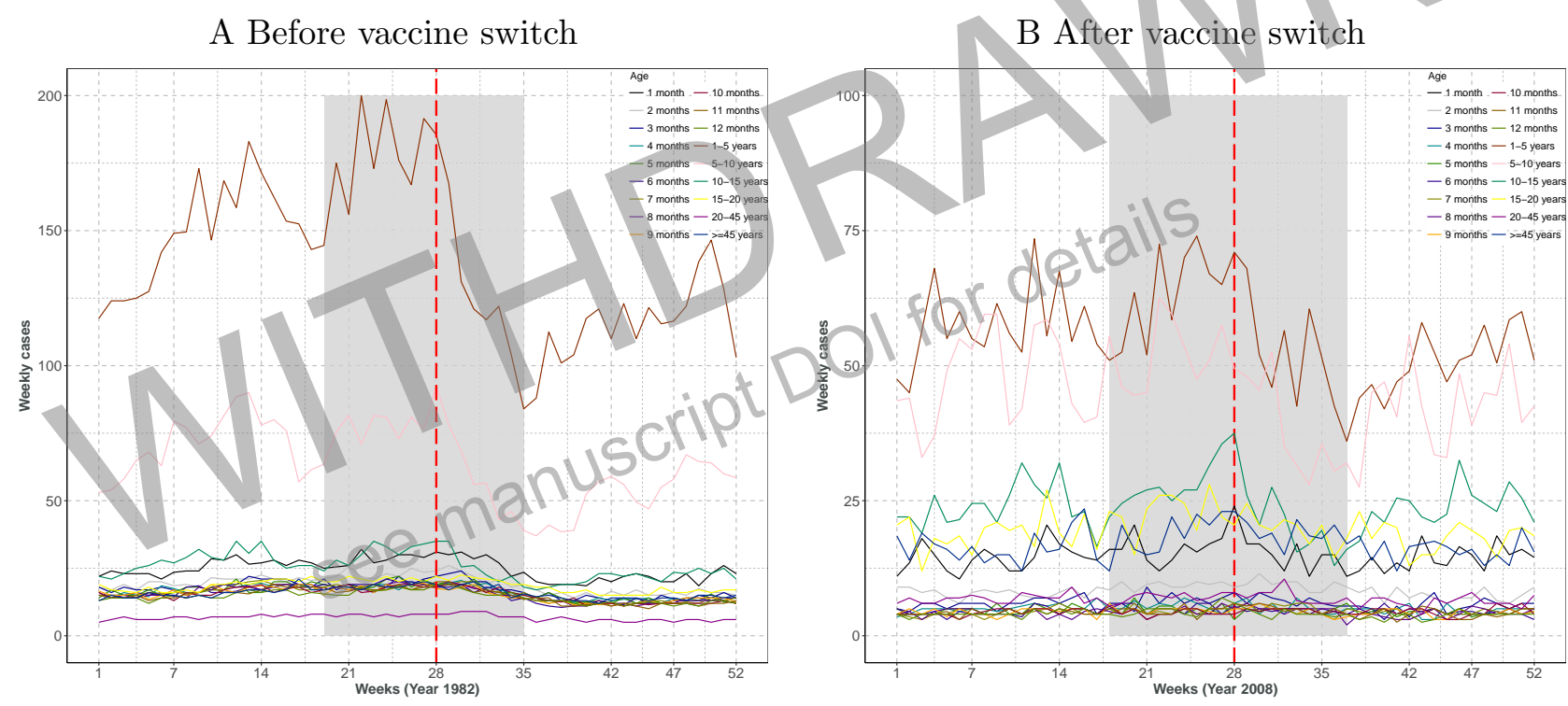

Figure 2: Simulated time series: Baseline scenario peak week - No changes in adult reporting, no asymptomatic transmission. Colors in the time series represent pertussis weekly incidence for each age class. Red vertical dashed line depicts peak week. Gray band encompasses ascending to peak week period and descending from peak week periods. A shows peak week in 1982, during the DTP period. B shows peak week after the change to DaTP in 2008 (4 years after DaTP replaced DTP). 


\section{Before the vaccine switch (DTP)}

Table 2: Odds ratios for each pair of age groups (rows vs columns) for the pre peak vs post peak periods and $95 \%$ confidence interval during a specific epidemic before the vaccine switch and increase adult reporting outbreak. Cells in light gray or red indicate that the confidence intervals do not contain 1. Light gray entries indicate that the age group in the row has a significantly higher relative risk of cases in the pre-peak period than the age group in the column, suggesting that the age group in the column is in some sense peaking earlier during the course of the epidemic. Red entries indicate that the relative risk of pre-peak cases is significantly lower for the age-group in the row.

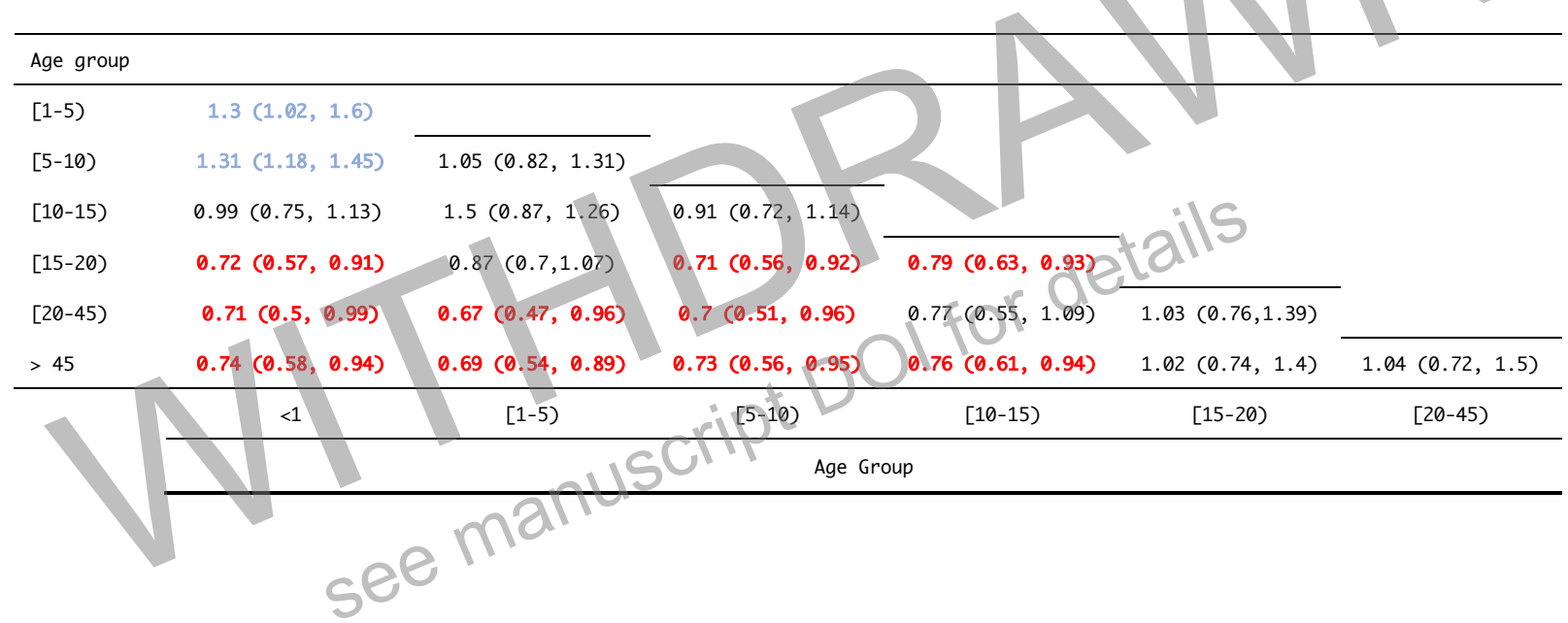

Table 2 provides a pairwise comparison of age groups, the odds ratios (OR), possibly indicating that young children (1-10-year-olds) were a key group in the epidemic. Worby et al.[21] described this higher risk as a measure for experiencing a significant depletion of susceptible pool during the outbreak take-off (pre-peak) period when compared to the other age groups, with risks among 1-10 years old significantly higher. 


\section{After the vaccine switch (4 years with DTaP)}

Table 3: Odds ratios for each pair of age groups (rows vs columns) for the pre peak vs post peak periods and $95 \%$ confidence interval during a specific epidemic after the vaccine switch (4 years after) and Increase adult reporting ( 7 years after) outbreak. Odds ratios for each pair of age groups (rows vs columns) for the pre peak vs post peak periods and $95 \%$ confidence interval during a specific epidemic before the vaccine switch and increase adult reporting outbreak. Cells in light gray or red indicate that the confidence intervals do not contain 1 . Light gray entries indicate that the age group in the row has a significantly higher relative risk of cases in the pre-peak period than the age group in the column, suggesting that the age group in the column is in some sense peaking earlier during the course of the epidemic. Red entries indicate that the relative risk of pre-peak cases is significantly lower for the age-group in the row.

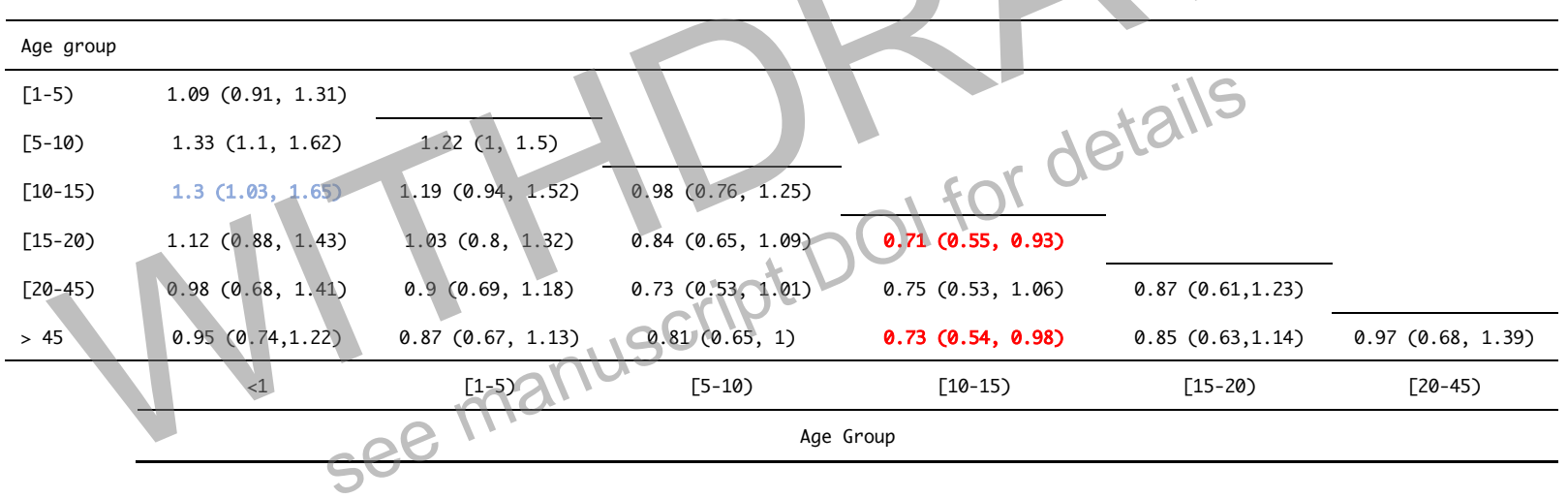

Table 3 provides a pairwise comparison of age groups, the odds ratios (OR), possibly indicating that young children (10-15-year-olds) were a key group in the epidemic. In 1982 the pre-peak relative risk of an age group was higher the closer that group was to ages 1-10 years old, suggesting that school-aged children were on the leading edge of the outbreak.

\section{Discussion}

\section{Needs updating}

1. Are the odds ratios used by Worby et al. [21] really a measure of susceptible depletion, as postulated by them? Or simply a feature of the fact of contacts shaping the transmission dynamics and also increase reporting?

2. Can asymptomatic carriage [17], as a consequence of introduction of acellular vaccine, explain he breakdown in signal of specific age groups? 
3. Can an increase in reporting probability of adults explain he breakdown in signal of specific age groups?

4. Determine by simulation how different scenarios affect the transmission dynamics and information regarding specific age groups

5. Calculate odds ratios and evaluate assumptions, in the context of contact rates

The relative impact of different age groups in epidemics in not well understood. We set out to test whether the observed changes in core groups could be due to asymptomatic transmission in infectious individuals who had been vaccinated with the DTaP vaccine (as suggested by $[22,17,10]$ ). We report the relative risks and odds ratio examining the impact of these differing assumptions on signatures of relative roles of key age groups through time. We assume that odds ratio are suggestive of driver role of a particular age group[21].

Our simulation study examined scenarios revealed that.

Scenario I- No asymptomatic transmission even after vaccination switch from DTP to DTaP (ie, both vaccines are transmission blocking). There are no changes to the adult reporting model (Figures 1 and 2).

Scenario II - Asymptomatic transmission can result following vaccination DTaP. We added a compartment to the baseline model: Infected Asymptomatic (A). Individuals who are vaccinated with Tdap may become infectious but will not show symptoms. There are no changes to the adult reporting model. This scenario with asymptomatic carriage (II), identified the 1-5 years of age as having a higher relative risk when compared to the other age groups (Figures S2 and S6 and Table S1).

Scenario III - No asymptomatic transmission in DTaP vaccinees. Increased adult reporting after the introduction of serological/PCR testing (Figures S3 and S7 and Table S2). The odds ratio results, for epidemics after the vaccine switch, that best reflect the loss of signal we observed in the actual data for England and Wales [36], are those for scenario III (increased adult reporting, no asymptomatic transmission).

Scenario IV - Both asymptomatic transmission after the vaccine switch and increased adult reporting after the introduction of serological/PCR testing. This scenario (both changes) shows age groups 1-10 years of age as being at higher risk, with 10-15 also with higher risk, possibly reflecting the roles these groups play in driving the epidemic (Figures S4 and S8 and Table S3).

Our results provide a cross-scenario comparison of the relative roles of different age cohorts during epidemics. Our estimates of the simulated results illustrate variability in the relative roles of each age 
group during different scenario pertussis epidemics, and for the most part yield consistent evidence for a leading role of school age children in propagating the pertussis epidemics.

\section{References}

[1] SA Plotkin and WA Orenstein. Vaccines. WB Saunders, Philadelphia, 3rd edition, 1999.

[2] N Wood and P Maclntyre. Pertussis: review of epidemiology, diagnosis, management and prevention. Pediatr Respir Rev, 8:291-212, 2008.

[3] Nicole Guiso. Bordetella pertussis and pertussis vaccines. Clinical Infectious Diseases, 49(10):15651569, 2009.

[4] Romina Libster and Kathryn $M$ Edwards. Re-emergence of pertussis: what are the solutions? Expert Rev Vaccines, 11(11):1331-46, Nov 2012.

[5] Pejman Rohani, David JD Earn, and Bryah T Grenfell. Opposite patterns of synchrony in sympatric disease metapopulations. Science, 286(5441):968-971, 1999.

[6] Pejman Rohani, David JD Earn, and Bryan T Grenfell. Impact of immunisation on pertussis transmission in England and Wales. The Lancet, 355(9200):285-286, 2000.

[7] H Campbell G Amirthalingam, S Gupta. Pertussis immunisation and control in england and wales, 1957 to 2012: a historical review. Eurosurveillance., 18:38, 2013.

[8] Maria A Riolo, Aaron A King, and Pejman Rohani. Can vaccine legacy explain the british pertussis resurgence? Vaccine, 31(49):5903-5908, 2013.

[9] WHO. Weekly epidemiological record. Pediatr Respir Rev, 89:221-236, 2014.

[10] Yoon H Choi, H Campbell, G Amirthalingam, A Jan van Hoek, and E Miller. Investigating the pertussis resurgence in england and wales, and options for future control. BMC Med, 14:121-132, 2016.

[11] AM Wendelboe, MG Hudgens, C Poole, and A Van Rie. Estimating the role of casual contact from the community in transmission of bordetella pertussis to young infants. Emerg. Themes Epidemiol., pages 4-15, 2007. 
[12] Ntezayabo B, De Serres G, and Duval B. Pertussis resurgence in canada largely caused by a cohort effect. Pediatr Infect Dis J., 22(1):22-27, 2003.

[13] J S Lavine and O N King, A A Bjornstad. Natural immune boosting in pertussis dynamics and the potential for long-term vaccine failure. Proceedings of the National Academy of Sciences, 108:7259?7264, 2011.

[14] N Wood and P Maclntyre. Pertussis in early infancy: disease burden and preventive strategies. Opin Infect Dis, 22:215-223, 2009.

[15] TM Skoff, C Kenyon, N Cocoros, J Liko, JL Miller, K Kudish, J Baumbach, S Zansky, A Faulkner, and SW Martin. Sources of infant pertussis infection in the united states. Pediatrics, 136(4):1-7, 2015.

[16] FMG Magpantay, MA Riolo, M Domenech de Celles, AA King, and P Rohani. Epidemiological consequences of imperfect vaccines for immunizing infections. SIAM Journal on Applied Mathematics, $74(6): 1810-1830,2014$.

[17] Benjamin M. Althouse and Samuel V. Scarpino. Asymptomatic transmission and the resurgence of bordetella pertussis. BMC Medicine, 13(1):146, 2015.

[18] JD Cherry. The science and fiction of the "resurgence" of pertussis. Pediatrics, 112(2):405-6, 2003.

[19] ED Shapiro. Acellular vaccines and resurgence of pertussis. JAMA, 308(20):2149-2150, 2012.

[20] Manoj Gambhir, Thomas A Clark, Simon Cauchemez, Sara Y Tartof, David L Swerdlow, and Neil M Ferguson. A change in vaccine efficacy and duration of protection explains recent rises in pertussis incidence in the United States. PLoS Comput Biol, 11(4):e1004138, Apr 2015.

[21] Colin J Worby, Cynthia Kenyon, Ruth Lynfield, Marc Lipsitch, and Edward Goldstein. Examining the role of different age groups, and of vaccination during the 2012 minnesota pertussis outbreak. Scientific reports, 5, 2015.

[22] Jason M Warfel, Lindsey I Zimmerman, and Tod J Merkel. Acellular pertussis vaccines protect against disease but fail to prevent infection and transmission in a nonhuman primate model. Proceedings of the National Academy of Sciences, 111(2):787-792, 2014. 
[23] John M Lachin. Biostatistical methods: the assessment of relative risks, volume 509. John Wiley \& Sons, 2011.

[24] HJ Wearing and $P$ Rohani. Estimating the duration of pertussis immunity using epidemiological signatures. PLoS Pathog, 5(10):e1000647, 2009.

[25] Pejman Rohani, Xue Zhong, and Aaron A King. Contact network structure explains the changing epidemiology of pertussis. Science, 330(6006):982-5, 2010.

[26] JC Blackwood, DAT Cummings, H Broutin, S lamsirithaworn, and P Rohani. Deciphering the impacts of vaccination and immunity on pertussis epidemiology in thailand. Proc Natl Acad Sci, 110(23):9595?600, 2010.

[27] Maria A Riolo and Pejman Rohani. Combating pertussis resurgence. One booster vaccination schedule does not fit all. Proceedings of the National Academy of Sciences, pages E472-E477, 2015.

[28] Joël Mossong, Niel Hens, Mark Jit, Philippe Beutels, Kari Auranen, Rafael Mikolajczyk, Marco Massari, Stefania Salmaso, Gianpaolo Scalia Tomba, Jacco Wallinga, Janneke Heijne, Malgorzata Sadkowska-Todys, Magdalena Rosinska, and W John Edmunds. Social contacts and mixing patterns relevant to the spread of infectious diseases. PLoS Med, 5(3):e74, Mar 2008.

[29] H Broutin, C Viboud, BT Grenfell, MA Miller, and P Rohani. Impact of vaccination and birth rate on the epidemiology of pertussis: A comparative study in 64 countries. Proc $R$ Soc Lond B Biol Sci, 282(1800):1?7, 2010.

[30] FMG Magpantay, M Domenech de Celles, AA King, and P Rohani. Pertussis immunity and epidemiology: mode and duration of vaccine-induced immunity. Parasitology, 4:1-15, 2015.

[31] PT Campbell, JM McCaw, P Mclntyre, and J McVernon. Defining long-term drivers of pertussis resurgence, and optimal vaccine control strategies. Vaccine, 33:5794?800, 2015.

[32] Matthieu Domenech de Celles, F M G Magpantay, Aaron A King, and Pejman Rohani. The pertussis enigma: Reconciling epidemiology, immunology, and evolution. Proc Roy Soc. B, 283:20152309, 2016.

[33] O Diekmann, J Heesterbeek, and M Roberts. The construction of next-generation matrices for compartmental epidemic models. J R Soc Interface, 7:873-885, 2010. 
[34] Simon Wood. Generalized Additive Models: An Introduction with R. CRC Press, 2006.

[35] Christian Ritz and Jens Carl Streibig. Nonlinear Regression with R. Springer, 2008.

[36] Bento Al, Riolo MA, Choi YH, King AA, and Rohani P. Core pertussis transmission groups in england and wales: A tale of two eras. In revision, 2018.

[37] Maria A Riolo, Aaron A King, and Pejman Rohani. Can vaccine legacy explain the British pertussis resurgence? Vaccine, 31(49):5903-8, Dec 2013.

[38] M J Keeling and P Rohani. Modeling diseases in Humans and Animals. Princeton University Press, 2008.

[39] M Kuhn and K Johnson. Applied Predictive Modeling. Springer, 2013. 


\section{Supplementary Information}

\section{Model formulation}

We implemented an age-structured, compartmental model of pertussis transmission, using previous described models as reference [1-3]. The model is an extension of the classic SIR model, it incorporates routine vaccination. Susceptibles individuals may become infected on contact with infected individuals. Infected individuals upon recovery move to the recovered class R. To account for possible differences between infection and vaccine-derived immunity, vaccinated individuals are explicitly modelled (V).

When modelling routine vaccination, for simplicity, we only consider one type of routine vaccination failure- Waning - where immunized individuals lose their immunity and become susceptible at a rate $\epsilon V$ for vaccine-derived immunity.

Overall, the model consists of 18 age groups, labelled $i=1, \ldots, 18$. The age groups are as follows, 12 1-month infant age classes and the following age categories for the older age groups (1-4), (5-19), (1014), (15-19), (20-44), 45+. We include the newborn classes to account for the maternal and routine immunization effects. Routine infant vaccination occurs at 2,4, and 6 mo of age in order to mimic the protective effects afforded following the receipt of three doses of pertussis vaccine. Aging occurs continuously, at rates $\alpha_{i}=\frac{1}{\Delta a_{i}} y r^{-1}$, where $\Delta a_{i}$ is the age span in age group i.

To model the effect of routine vaccination, a fraction of the individuals either from $\mathrm{S}$ are moved to $V$ on aging from 1 to 2 months of age.

The model is described by the system of differential equations below and was integrated numerically. 


\section{Model equations}

For newborns up to 1 month old $(i=1)$ :

$$
\begin{aligned}
\frac{d V_{1}}{d t} & =0 \\
\frac{d A_{1}}{d t} & =0 \\
\frac{d S_{1}}{d t} & =\nu N-\left(\lambda_{1}-\mu 1-\alpha 1\right) S_{1}+\epsilon_{1} R_{1} \\
\frac{d I_{1}}{d t} & =\lambda_{1} S_{1}-\left(\gamma-\alpha_{1}-\mu_{1}\right) I_{1} \\
\frac{d R_{1}}{d t} & =\gamma I_{1}-\left(\alpha_{1}+\epsilon_{1}+\mu_{1}\right) R_{1}
\end{aligned}
$$

For infants age 2 - 6 months old $(i=2,4,6)$ :

$$
\begin{aligned}
& \frac{d V_{i}}{d t}=-\left(\epsilon+\mu_{i}+\alpha_{i}\right) V_{i}+\left(\alpha_{i-1} S_{i}\right) \rho \\
& \frac{d A_{i}}{d t}=\lambda_{i} V_{i}-\left(\gamma-\mu_{i}\left(\alpha_{i}\right) A_{i}+\alpha_{i-1} A_{i-1}\right. \\
& \frac{d S_{i}}{d t}=\left(\lambda_{i} 2 \mu_{i}-\alpha_{i}\right) S_{i}+\epsilon_{i} R_{i}-\epsilon_{i} V_{i}+\left(\alpha_{i-1} S_{i-1}\right)(1-\rho) \\
& \frac{d I_{i}}{d t}=\lambda_{i} S_{i}-\left(\gamma-\alpha_{i}-\mu_{i}\right) I_{i}+\alpha_{i-1} I_{i-1} \\
& \frac{d R_{i}}{d t}=\gamma I_{i}-\left(\alpha_{i}+\epsilon_{i}+\mu_{i}\right) R_{1}+\alpha_{i-1} R_{i-1}
\end{aligned}
$$

For infants age $3,5,7$ months old to $45+(i=3,5,7 \ldots, 18)$ :

$$
\begin{aligned}
\frac{d V_{i}}{d t} & =-\left(\epsilon+\mu_{i}+\alpha_{i}\right) V_{i}+\alpha_{i-1} V_{i-1} \\
\frac{d A_{i}}{d t} & =\lambda_{i} V_{i}-\left(\gamma-\mu_{i}-\alpha_{i}\right) A_{i}+\alpha_{i-1} A_{i-1} \\
\frac{d S_{i}}{d t} & =\left(\lambda_{i}-\mu_{i}-\alpha_{i}\right) S_{i}+\epsilon_{i} R_{i}-\epsilon_{i} V_{i}+\alpha_{i-1} S_{i-1} \\
\frac{d I_{i}}{d t} & =\lambda_{i} S_{i}-\left(\gamma-\alpha_{i}-\mu_{i}\right) I_{i}+\alpha_{i-1} I_{i-1} \\
\frac{d R_{i}}{d t} & =\gamma I_{i}-\left(\alpha_{i}+\epsilon_{i}+\mu_{i}\right) R_{1}+\alpha_{i-1} R_{i-1}
\end{aligned}
$$

The force of infection in age class $i$ is expressed as:

$$
\lambda_{i}=\sum_{j} q_{i}^{1} q_{i}^{2} F_{i j}(t) \frac{I_{j}^{1}+\theta I_{j}^{2}}{N_{j}}
$$


Here, q represents the probability of infection given contact, $C_{i j}$ the matrix of age-specific contact rates, and $S_{i j}(t)$ an age-specific seasonal forcing. Following ([37]), we assume seasonality only when a contact involves school age children ( 5 to 20 years old). Thus,

$$
S_{i j}(t) \begin{cases}\frac{1+\beta_{1} \operatorname{Term}(t)}{1+\frac{\beta_{1}}{365}(D+-D-)} & i \text { or } j \in 14,15,16 \\ 1, & \text { otherwise }\end{cases}
$$

with

$$
\operatorname{Term}(t)= \begin{cases}+1 & \text { during school term } \\ -1, & \text { during hollidays }\end{cases}
$$

the term-time forcing function, D the total number of days of school, and D- the total number of days of holidays. The denominator in the equation ensures that: $\forall(i, j) ; \mathbb{E}_{t}\left[S_{i j}(t)\right]=1 \quad([38])$

\section{S7.2 Next generation matrix and $R_{0}$}

Our model used the Great Britain empirical age-specific contact rates from the POLYMOD study [28], corrected for reciprocity [377. Where, $C_{i j}$ is the average number of daily contacts in Mossong et al. [28] reported by a "contacter" of age group $i$ with "contactees" of age group $j$ (individuals are classified into 0-1 year and then (1-4), (5-19), (10-14), (15-19), (20-44), 45+ age groups so that $1 \leq(i, j) \leq 18)$. $N_{i}$ represents individuals in age group $\mathrm{i}$ in the population. $E_{i j}=N_{i} C_{i j}$. is the average total number of contacts between age groups $\mathrm{i}$ and $\mathrm{j}$. The matrix $\mathrm{E}=(\mathrm{Eij})$ was transformed to become symmetric: $\mathrm{E} \rightarrow \frac{1}{2}\left(E+E^{T}\right)$. Thus, the individual average number of daily contacts between age groups $i$ and $j$, corrected for reciprocity: $\forall(i, j), C i j=\frac{E_{i j}}{N_{i}}$. We augmented the age class $0-1$ years of age into monthly classes by replicating the (0-1 years) 12 times making sure the sum of contacts is the same as before. We assumed that individuals in the 01 years age group have identical number of contacts: $\forall(j)$, augmented $C_{1 j}=$ augmented $C_{2 j}=\ldots$ augmented $C_{12 j}=C_{1 j} . \quad R_{0}$ is the basic reproduction number in the absence of vaccination $(\rho=0)$. Calculations used the age classes defined above $(i=1$, $\ldots, 18)$ and a constant birth rate $\nu=\frac{1}{75} y r^{-} 1$, at disease free equilibrium in the absence of vaccination $(\rho=0): \forall_{i}, S_{i}=N_{i}, V_{i}=0$, where $R_{i}=0$ is the dominant eigenvalue of the next-generation matrix [? ]. School-aged children have the highest total contact rates, followed by 35- to 45-year-olds. However, the contacts of 35- to 45-year-olds are spread among a wide range of age groups, whereas mixing among school-aged children is very strongly age-assortative [28]. Thus, although infections in school-aged children and 35- to 45-year-olds both generate a relatively large number of new infections, 
bioRxiv preprint doi: https://doi.org/10.1101/247007; this version posted January 11,2018 . The copyright holder for this preprint (which was not certified by peer review) is the author/funder. All rights reserved. No reuse allowed without permission.

Relative role of age groups

school-aged children tend to infect children their own age, who also have high contact rates, whereas the infectious contacts of 35- to 45-y- olds will include a wide range of age groups with varying frequency of contacts.

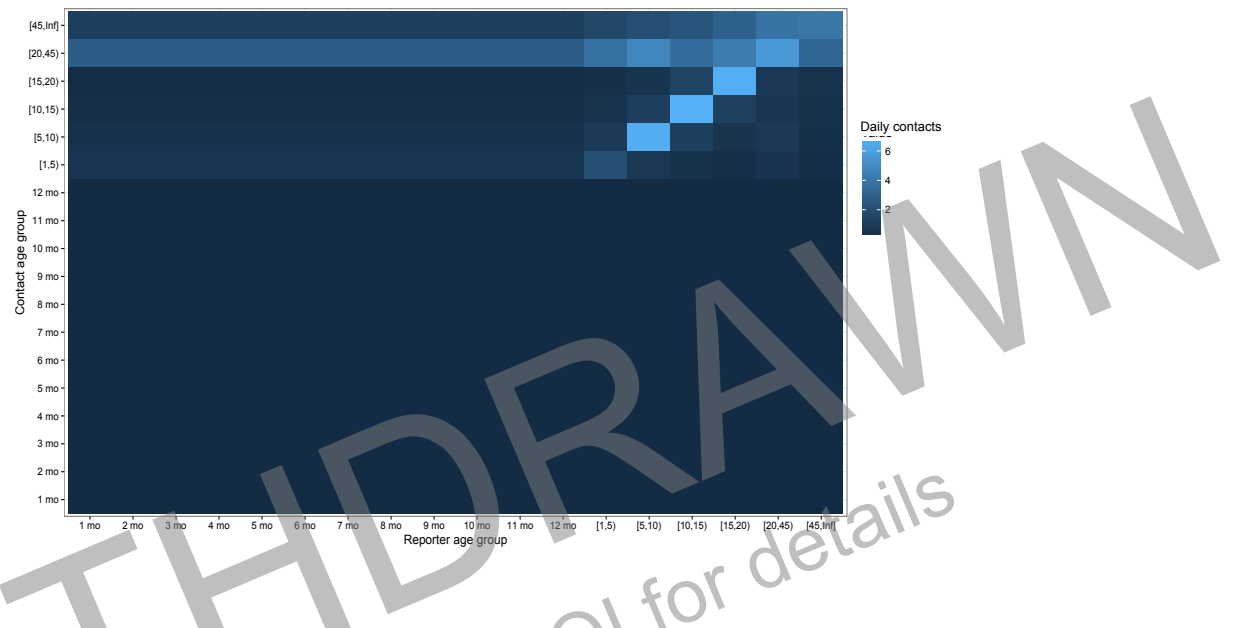

Figure S1: Age-specific contact matrix used in the simulations.

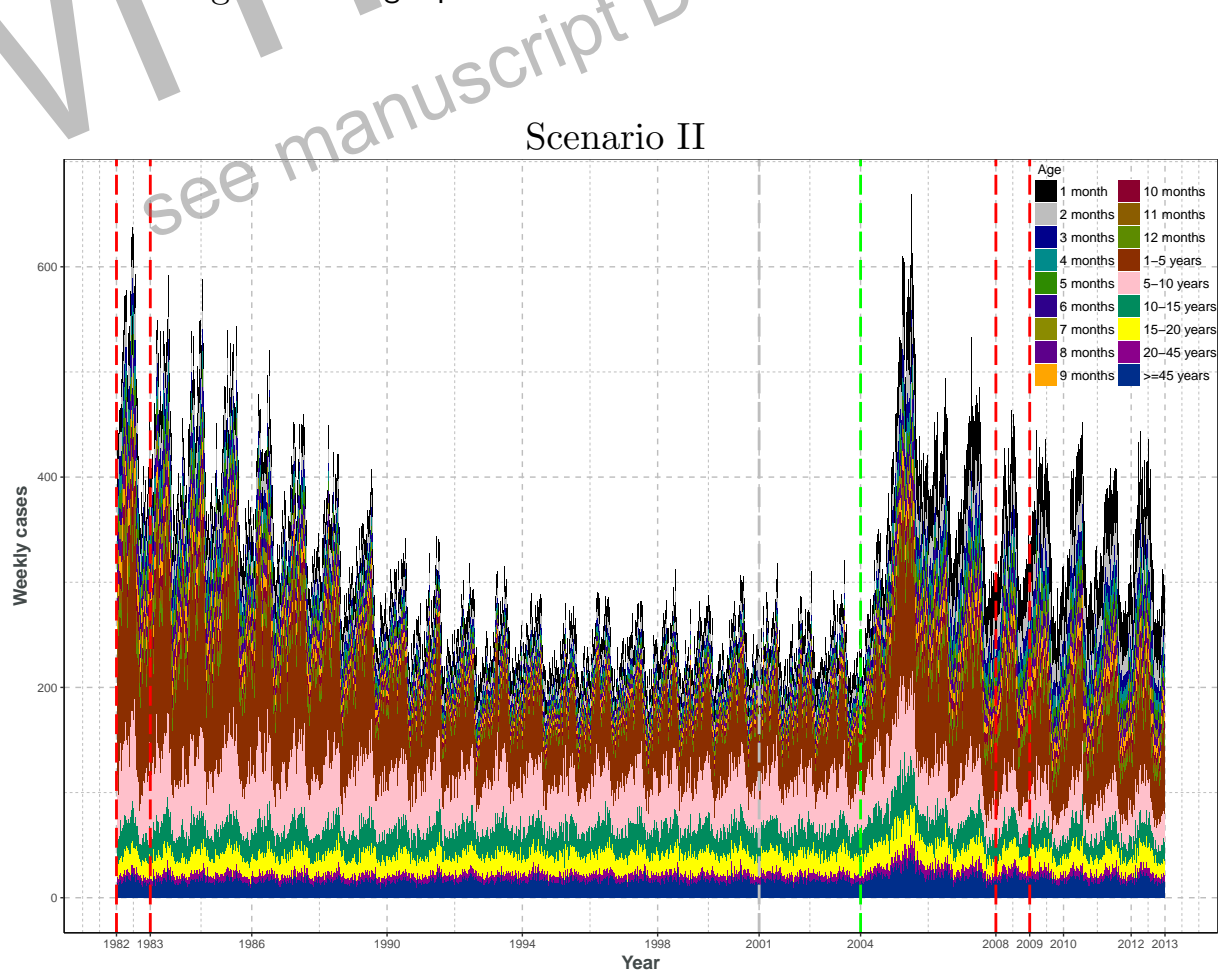

Figure S2: Simulated time series: Scenario II - No changes in adult reporting, with asymptomatic transmission (DaTP vaccinated individuals that become infected). Colors in the time series represent pertussis weekly incidence for each age class. Red vertical dashed lines encompass the epidemic years (1982 and 2008) used for the odds ratio calculations. Grey vertical dashed line shows year (2001) of changes in reporting (Scenario II does not simulate increase in adult reporting). Green vertical dashed line represents the year DTP was replaced by DaTP (2004). 
bioRxiv preprint doi: https://doi.org/10.1101/247007; this version posted January 11,2018 . The copyright holder for this preprint (which was not certified by peer review) is the author/funder. All rights reserved. No reuse allowed without permission.

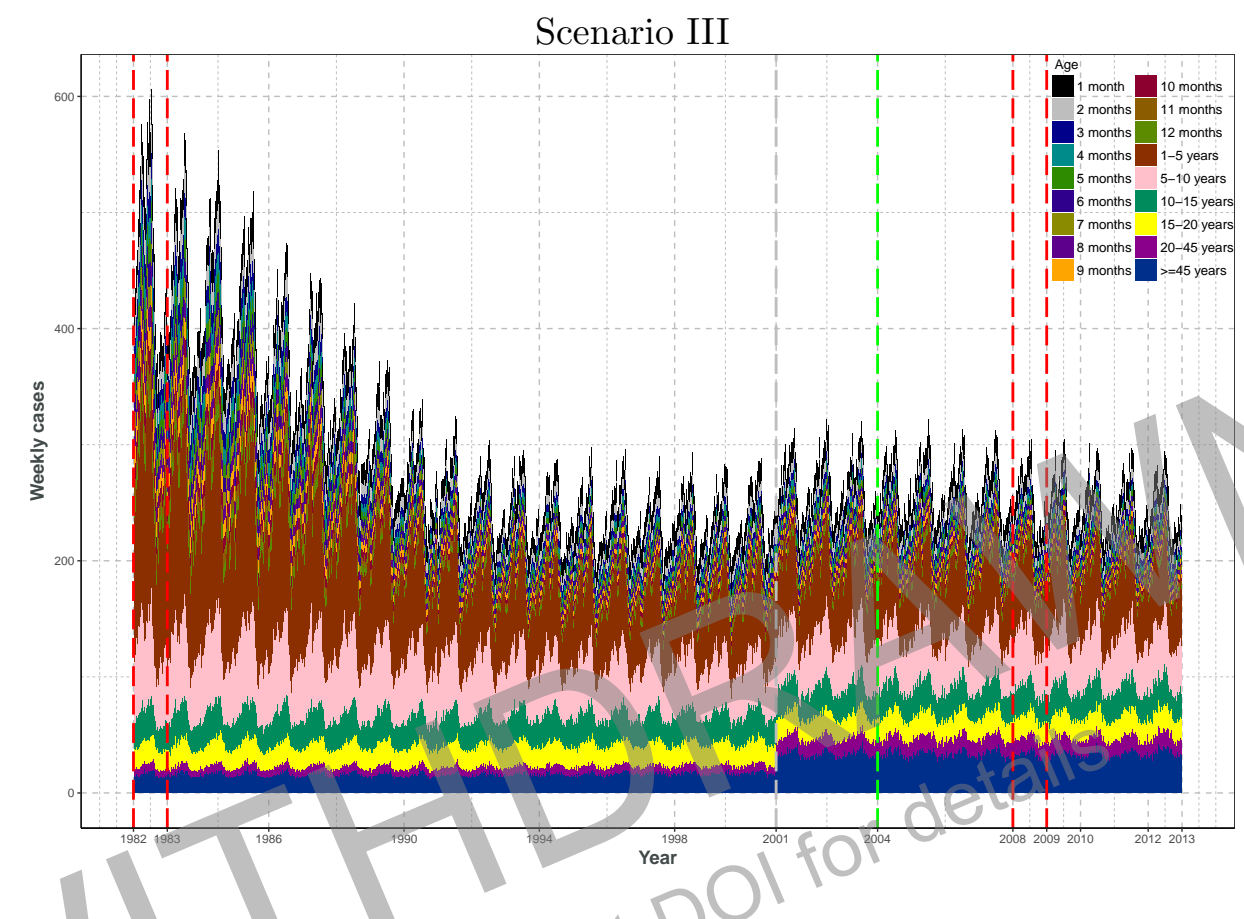

Figure S3: Simulated time series: Scenario III - Changes in adult reporting, with no asymptomatic transmission (DaTP vaccinated individuals that become infected). Colors in the time series represent pertussis weekly incidence for each age class. Red vertical dashed lines encompass the epidemic years (1982 and 2008) used for the odds ratio calculations. Grey vertical dashed line shows year (2001) of changes in reporting (Scenario II does not simulate increase in adult reporting). Green vertical dashed line represents the year DTP was replaced by DaTP (2004). 


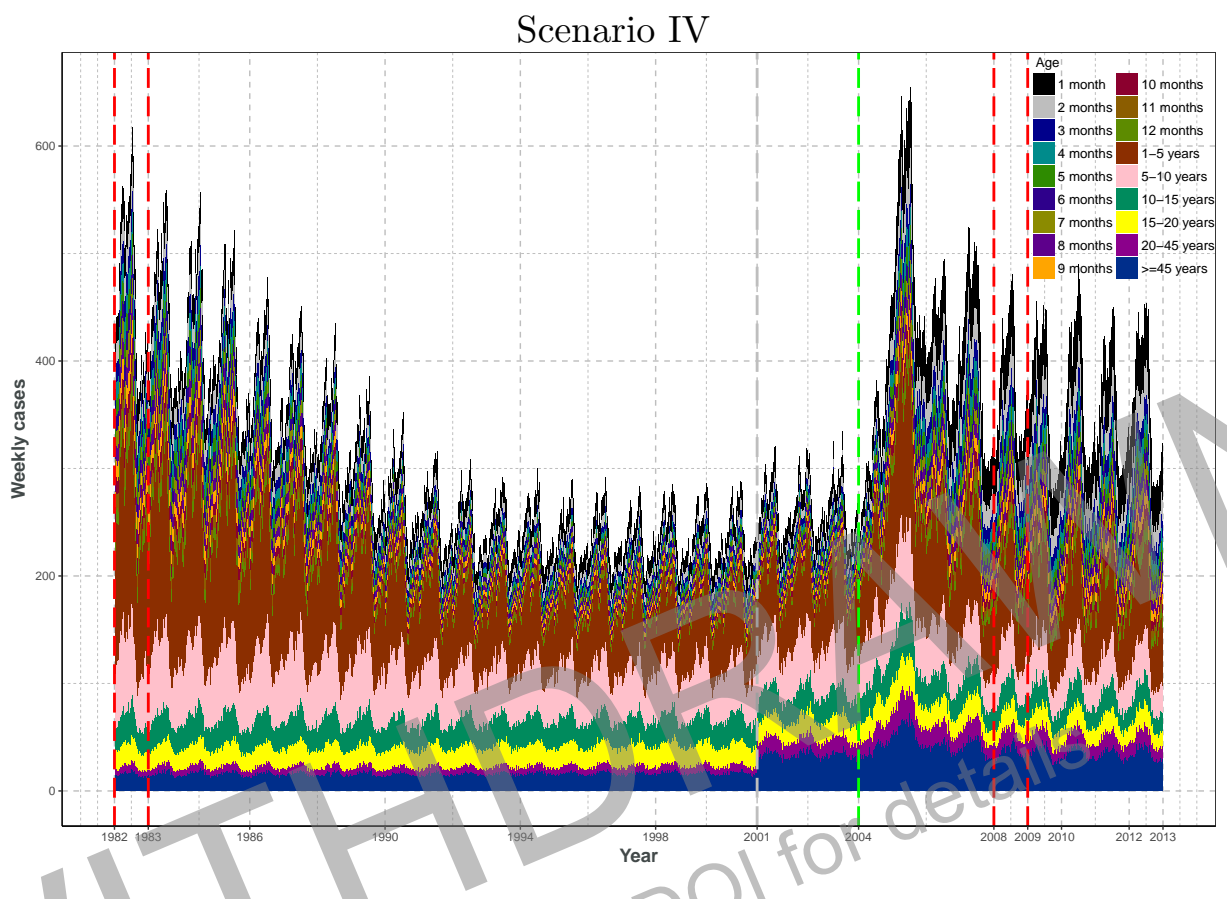

Figure S4: Simulated time series: Scenario IV - Changes in adult reporting, with asymptomatic transmission (DaTP vaccinated individuals that become infected). Colors in the time series represent pertussis weekly incidence for each age class. Red vertical dashed lines encompass the epidemic years (1982 and 2008) used for the odds ratio calculations. Grey vertical dashed line shows year (2001) of changes in reporting (Scenario II does not simulate increase in adult reporting). Green vertical dashed line represents the year DTP was replaced by DaTP (2004).

\section{Odds ratios}

We defined the peak week, as the calendar week, in a given epidemic year, with the highest total number of recorded cases. The pre peak period was by a general additive model (GAM). The period post peak week was determined by the same method using the cut off week with the emergence of a smaller peak in incidence. An example year is shown. 
bioRxiv preprint doi: https://doi.org/10.1101/247007; this version posted January 11,2018 . The copyright holder for this preprint (which was not certified by peer review) is the author/funder. All rights reserved. No reuse allowed without permission.

Relative role of age groups

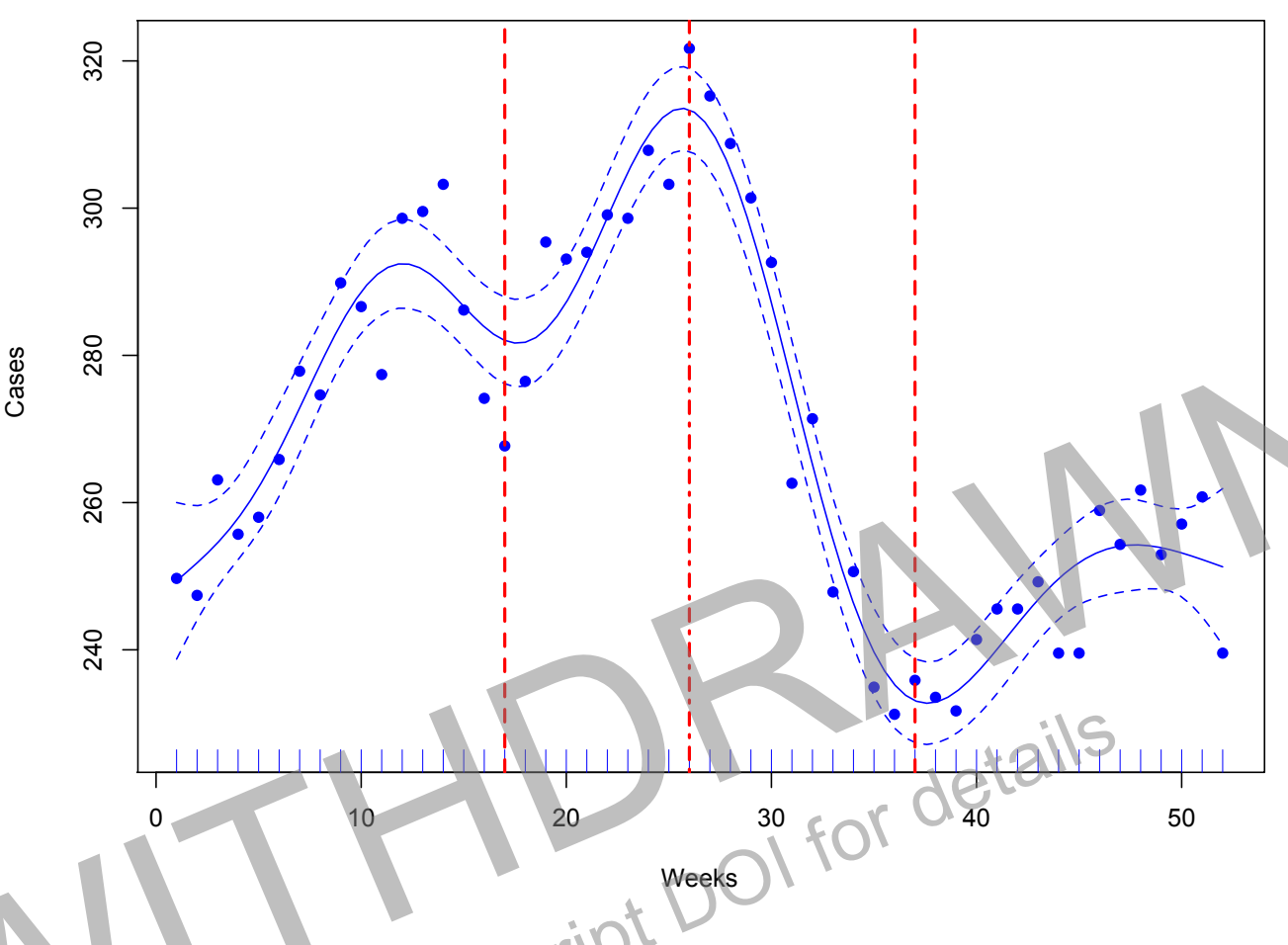

Figure S5: Periods estimation using GAM. Estimation of the pre peak period, peak week and post peak period for a given epidemic year. Red vertical dashed lines represent the peak week and interval between; ascending and peak; peak and descending 
The scenarios presented here are for after introduction of DTaP and after increase adult reporting.

\section{Scenario II: Asymptomatic carriage}

Figure S6: Simulated time series: Scenario II Peak Week No changes in adult reporting, with asymptomatic transmission after the change from DTP to DaTP after the change to DaTP in 2008 (4 years after DaTP replaced DTP). Colors in the time series represent pertussis weekly incidence for each age class. Red vertical dashed line depicts peak week. Gray band encompasses ascending to peak week period and descending from peak week periods.

Table S1: Odds ratios for each pair of age groups (rows vs columns) for the pre peak vs post peak periods and $95 \%$ confidence interval during a specific epidemic after the vaccine switch and Increase adult reporting outbreak. Cells in gray or red indicate that the confidence intervals do not contain 1.

\begin{tabular}{|c|c|c|c|c|c|c|}
\hline \multicolumn{7}{|c|}{ Age group } \\
\hline$[1-5)$ & $1.81(1.24,2.65)$ & & & & & \\
\hline$[5-10)$ & $1.06(0.74,1.52)$ & $0.58(0.38,0.9)$ & & & & \\
\hline$[10-15)$ & $0.97(0.62,1.52)$ & $0.54(0.32,0.89)$ & $0.92(0.56,1.51)$ & & & \\
\hline$[15-20)$ & $0.69(0.44,1.07)$ & $0.38(0.23,0.63)$ & $0.65(0.4,1.06)$ & $0.71(0.55,0.93)$ & & \\
\hline$[20-45)$ & $0.92(0.45,1.89)$ & $0.51(0.24,1.08)$ & $0.87(0.55,1.38)$ & $0.71(0.41,1.23)$ & $1.33(0.79,2.23)$ & \\
\hline \multirow[t]{2}{*}{$>45$} & $0.26(0.74,2.13)$ & $0.69(0.39,1.24)$ & $1.19(0.68,2.1)$ & $1.29(0.69,2.41)$ & $1.83(0.99,3.39)$ & $1.37(0.75,2.5)$ \\
\hline & $<1$ & {$[1-5)$} & {$[5-10)$} & {$[10-15)$} & {$[15-20)$} & {$[20-45)$} \\
\hline
\end{tabular}




\section{Scenario III: Increase adult reporting}

Figure S7: Simulated time series: Scenario III Peak Week - Changes in adult reporting, with no asymptomatic transmission after the change from DTP to DaTP after the change to DaTP in 2008 (4 years after DaTP replaced DTP). Colors in the time series represent pertussis weekly incidence for each age class. Red vertical dashed line depicts peak week. Gray band encompasses ascending to peak week period and descending from peak week periods.

Table S2: Odds ratios for each pair of age groups (rows vs columns) for the pre peak vs post peak periods and $95 \%$ confidence interval during a specific epidemic after the vaccine switch and Increase adult reporting outbreak. Cells in gray or red indicate that the confidence intervals do not contain 1.

\begin{tabular}{|c|c|c|c|c|c|c|}
\hline \multicolumn{7}{|c|}{ Age group } \\
\hline$[1-5)$ & $1.11(0.93,1.32)$ & & & & & \\
\hline$[5-10)$ & $1.75(1.53,2.01)$ & $1.09(0.9,1.32)$ & & & & \\
\hline$[10-15)$ & $1.12(1,1.45)$ & $1.02(0.82,1.28)$ & $0.94(0.74,1.19)$ & & & \\
\hline$[15-20)$ & $1(0.79,1.26)$ & $0.9(0.71,1.15)$ & $0.83(0.64,1.06)$ & $0.88(0.67,1.16)$ & & \\
\hline$[20-45)$ & $1.31(1.11,1.56)$ & $0.9(0.69,1.18)$ & $0.78(0.65,1.05)$ & $0.88(0.68,1.15)$ & $1(0.76,1.32)$ & \\
\hline \multirow[t]{2}{*}{$>45$} & $1(0.76,1.3)$ & $0.88(0.72,1.08)$ & $0.81(0.65,1)$ & $0.86(0.67,1.1)$ & $0.98(0.75,1.27)$ & $0.98(0.76,1.19)$ \\
\hline & $<1$ & {$[1-5)$} & {$[5-10)$} & {$[10-15)$} & {$[15-20)$} & {$[20-45)$} \\
\hline
\end{tabular}




\section{Scenario IV: Both II and III}

Figure S8: Simulated time series: Scenario IV Peak Week - Changes in adult reporting, with asymptomatic transmission after the change from DTP to DaTP after the change to DaTP in 2008 (4 years after DaTP replaced DTP). Colors in the time series represent pertussis weekly incidence for each age class. Red vertical dashed line depicts peak week. Gray band encompasses ascending to peak week period and descending from peak week periods.

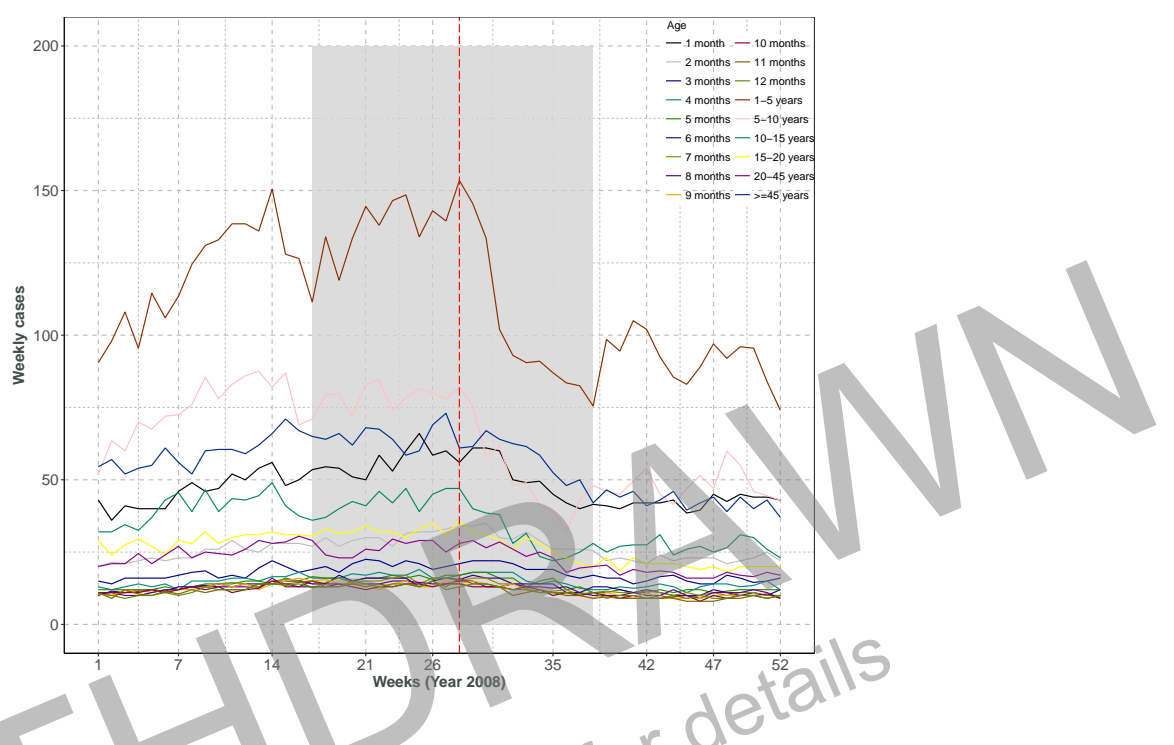

Table S3: Odds ratios for each pair of age groups (rows vs columns) for the pre peak vs post peak periods and $95 \%$ confidence interval during a specific epidemic after the vaccine switch and Increase adult reporting outbreak. Cells in gray or red indicate that the confidence intervals do not contain 1.

\begin{tabular}{|c|c|c|c|c|c|c|}
\hline \multicolumn{7}{|c|}{ Age group } \\
\hline$[1-5)$ & $1.23(1.26,1.68)$ & & & & & \\
\hline$[5-10)$ & $1.45(1.26,1.68)$ & $1.18(1.01,1.37)$ & & & & \\
\hline$[10-15)$ & $1.29(1.08,1.54)$ & $1.05(0.87,1.26)$ & $0.89(0.73,1.09)$ & & & \\
\hline$[15-20)$ & $1.08(0.9,1.3)$ & $0.87(0.72,1.06)$ & $0.74(0.6,0.92)$ & $0.84(0.66,1.06)$ & & \\
\hline$[20-45)$ & $0.99(0.82,1.21)$ & $0.81(0.66,0.99)$ & $0.76(0.61,0.95)$ & $0.77(0.63,0.95)$ & $0.92(0.74,1.14)$ & \\
\hline \multirow[t]{2}{*}{$>45$} & $1.03(0.89,1.19)$ & $0.83(0.72,0.97)$ & $0.68(0.57,0.82)$ & $0.8(0.65,0.97)$ & $0.95(0.77,1.18)$ & $1.04(0.86,1.24)$ \\
\hline & $<1$ & {$[1-5)$} & {$[5-10)$} & {$[10-15)$} & {$[15-20)$} & {$[20-45)$} \\
\hline
\end{tabular}




\section{Downsampling}

Because case reports in many age groups were relatively sparse, we binned weekly case data by age (starting from the youngest group, $<1$ month olds), such that, at least half of the weeks in each age bin have a non-zero number of cases, resulting in age bins that are increasingly coarse with age.

In order to disentangle the effects of lower overall case counts from underlying transmission patterns, we repeated our analyses on subsets of out case data downsampled such that we would expect the highest incidence peak in each (binned) age category to be equal [39].

We generated such a downsampled data set as follows. For each age group, we find the maximum incidence in a single week, Peak age, and designate the lowest of these (i.e. the lowest peak incidence among age groups) as Peak $k_{\min }$. Then, for each age group, we sample each week's case number from a

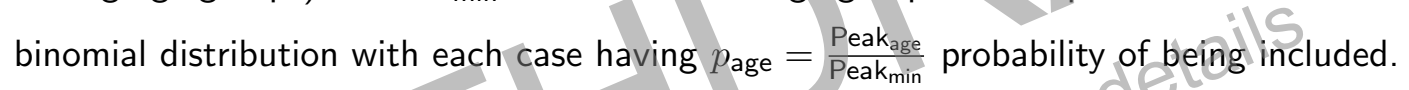

We generated one hundred such downsampled runs per scenario and performed odds ratio analyses on each run.

Same method, as with the raw data, with separate analysis of odds ratios for 1982 and 2008, using 100 subsampled time series. Like with the previous methods analysing downsampled data, relative risk and odds ratio analyses were not robust to downsampling, as the changes in sampling amplify the number of reported cases for those age groups that had fewer cases reported in the raw data. Thus, underestimating the risks for age groups that nonetheless were found to carry the highest estimates of the RR statistic, specifically for the 1982 epidemic. 


\section{Scenario I - Baseline: Before vaccine change}

Figure S9: Downsampled time series. Scenario I: Baseline before vaccine changes - No changes in adult reporting, and no asymptomatic transmission. Colors in the time series represent pertussis weekly incidence for each age class. Red vertical dashed line depicts peak week. Gray band encompasses ascending to peak week period and descending from peak week periods.

Table S4: Odds ratios for each pair of age groups (rows vs columns) for the pre peak vs post peak periods and $95 \%$ confidence interval during the 2012 national outbreak using 100 subsamplings of the data. Cells in gray or red indicate that the confidence intervals do not contain 1. Light gray entries indicate that the age group in the row has a significantly higher relative risk of cases in the pre-peak period than the age group in the column, suggesting that the age group in the column is in some sense peaking earlier during the course of the epidemic. Red entries indicate that the relative risk of pre-peak cases is significantly lower for the age-group in the row.

\begin{tabular}{|c|c|c|c|c|c|c|}
\hline Age group & & & & & & \\
\hline$[1-5)$ & $2 / 100$ & & & & & \\
\hline$[5-10)$ & $7 / 100$ & $100 / 100$ & & & & \\
\hline$[10-15)$ & $100 / 100$ & $100 / 100$ & $100 / 100$ & & & \\
\hline$[15-20)$ & $1 / 100$ & $100 / 100$ & $19 / 100$ & $7 / 100$ & & \\
\hline$[20-45)$ & $1 / 100$ & $2 / 100$ & $11 / 100$ & $1 / 100$ & $1 / 100$ & \\
\hline \multirow[t]{3}{*}{$>45$} & $10 / 100$ & $1 / 100$ & $16 / 100$ & $8 / 100$ & $100 / 100$ & $100 / 100$ \\
\hline & $<1$ & {$[1-5)$} & {$[5-10)$} & {$[10-15)$} & {$[15-20)$} & {$[20-45)$} \\
\hline & \multicolumn{6}{|c|}{ Age Group } \\
\hline
\end{tabular}




\section{Scenario I - Baseline: After vaccine change}

Figure S10: Downsampled time series. Scenario Baseline after vaccine change - No changes in adult reporting, and no asymptomatic transmission. Colors in the time series represent pertussis weekly incidence for each age class. Red vertical dashed line depicts peak week. Gray band encompasses ascending to peak week period and descending from peak week periods.

Table S5: Odds ratios for each pair of age groups (rows vs columns) for the pre peak vs post peak periods and $95 \%$ confidence interval during the 2012 national outbreak using 100 subsamplings of the data. Cells in gray or red indicate that the confidence intervals do not contain 1. Light gray entries indicate that the age group in the row has a significantly higher relative risk of cases in the pre-peak period than the age group in the column, suggesting that the age group in the column is in some sense peaking earlier during the course of the epidemic. Red entries indicate that the relative risk of pre-peak cases is significantly lower for the age-group in the row.

\begin{tabular}{|c|c|c|c|c|c|c|}
\hline \multicolumn{7}{|c|}{ Age group } \\
\hline$[1-5)$ & $100 / 100$ & & & & & \\
\hline$[5-10)$ & $1 / 100$ & $100 / 100$ & & & & \\
\hline$[10-15)$ & $1 / 100$ & $100 / 100$ & $1 / 100$ & & & \\
\hline$[15-20)$ & $100 / 100$ & $100 / 100$ & $1 / 100$ & $1 / 100$ & & \\
\hline$[20-45)$ & $100 / 100$ & $100 / 100$ & $1 / 100$ & $100 / 100$ & $100 / 100$ & \\
\hline$>45$ & $3 / 100$ & $100 / 100$ & $2 / 100$ & $2 / 100$ & $1 / 100$ & $2 / 100$ \\
\hline & $<1$ & {$[1-5)$} & {$[5-10)$} & {$[10-15)$} & {$[15-20)$} & {$[20-45)$} \\
\hline
\end{tabular}




\section{Scenario II - Asymptomatic carriage}

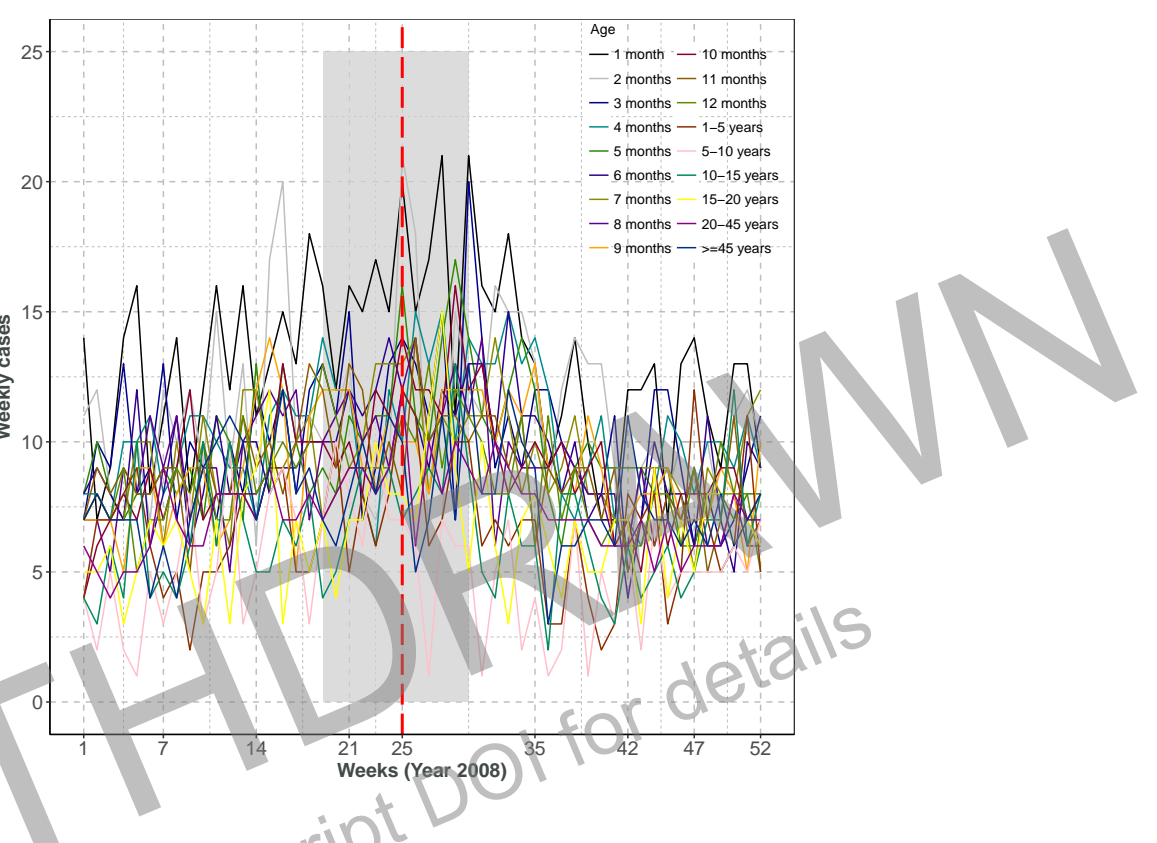

Figure S11: Downsampled time series. Scenario II- No changes in adult reporting, with asymptomatic transmission after the change from DTP to DaTP. Colors in the time series represent pertussis weekly incidence for each age class. Red vertical dashed line depicts peakweek. Gray band encompasses ascending to peak week period and descending from peak week periods.

Table S6: Odds ratios for each pair of age groups (rows vs columns) for the pre peak vs post peak periods and $95 \%$ confidence interval during the 2012 national outbreak using 100 subsamplings of the data. Cells in gray or red indicate that the confidence intervals do not contain 1. Light gray entries indicate that the age group in the row has a significantly higher relative risk of cases in the pre-peak period than the age group in the column, suggesting that the age group in the column is in some sense peaking earlier during the course of the epidemic. Red entries indicate that the relative risk of pre-peak cases is significantly lower for the age-group in the row.

\begin{tabular}{|c|c|c|c|c|c|c|}
\hline \\
\hline \multicolumn{7}{|l|}{$\begin{array}{l}\text { Age group } \\
{[1-5)}\end{array}$} \\
\hline$[5-10)$ & $11 / 100$ & $100 / 100$ & & & & \\
\hline$[10-15)$ & $100 / 100$ & $100 / 100$ & $100 / 100$ & & & \\
\hline$[15-20)$ & $5 / 100$ & $100 / 100$ & $10 / 100$ & $4 / 100$ & & \\
\hline$[20-45)$ & $9 / 100$ & $14 / 100$ & $21 / 100$ & $9 / 100$ & $7 / 100$ & \\
\hline \multirow[t]{2}{*}{$>45$} & $12 / 100$ & $6 / 100$ & $7 / 100$ & $12 / 100$ & $2 / 100$ & $100 / 100$ \\
\hline & $<1$ & {$[1-5)$} & {$[5-10)$} & {$[10-15)$} & {$[15-20)$} & {$[20-45)$} \\
\hline
\end{tabular}




\section{Scenario III- Increase adult reporting}

Figure S12: Downsampled time series. Scenario III - Changes in adult reporting, with no asymptomatic transmission after the change from DTP to DaTP. Colors in the time series represent pertussis weekly incidence for each age class. Red vertical dashed line depicts peak week. Gray band encompasses ascending to peak week period and descending from peak week periods.

Table S7: Odds ratios for each pair of age groups (rows vs columns) for the pre peak vs post peak periods and $95 \%$ confidence interval during the 2012 national outbreak using 100 subsamplings of the data. Cells in gray or red indicate that the confidence intervals do not contain 1 . Light gray entries indicate that the age group in the row has a significantly higher relative risk of cases in the pre-peak period than the age group in the column, suggesting that the age group in the column is in some sense peaking earlier during the course of the epidemic. Red entries indicate that the relative risk of pre-peak cases is significantly lower for the age-group in the row.

\begin{tabular}{lccccc}
\hline Age group & & & & & \\
\hline$[1-5)$ & $100 / 100$ & & & & \\
{$[5-10)$} & $1 / 100$ & $6 / 100$ & $100 / 100$ & & \\
{$[10-15)$} & $100 / 100$ & $100 / 100$ & $2 / 100$ & $4 / 100$ & $4 / 100$ \\
{$[15-20)$} & $7 / 100$ & $3 / 100$ & $1 / 100$ & $1 / 100$ & $3 / 100$ \\
{$[20-45)$} & $100 / 100$ & $2 / 100$ & $2 / 100$ & $100 / 100$ & {$[15-20)$} \\
$>45$ & $100 / 100$ & $100 / 100$ & {$[5-10)$} & {$[10-15)$} & {$[20-45)$} \\
\hline & $<1$ & {$[1-5)$} & & Age Group & \\
\cline { 2 - 5 }
\end{tabular}




\section{Scenario IV - Both II and III}

Figure S13: Downsampled time series. Scenario IV, Changes in adult reporting, with asymptomatic transmission after the change from DTP to DaTP. Colors in the time series represent pertussis weekly incidence for each age class. Red vertical dashed line depicts peakweek. Gray band encompasses ascending to peak week period and descending from peak week periods.

Table S8: Odds ratios for each pair of age groups (rows vs columns) for the pre peak vs post peak periods and $95 \%$ confidence interval during the 2012 national outbreak using 100 subsamplings of the data. Cells in gray or red indicate that the confidence intervals do not contain 1 . Light gray entries indicate that the age group in the row has a significantly higher relative risk of cases in the pre-peak period than the age group in the column, suggesting that the age group in the column is in some sense peaking earlier during the course of the epidemic. Red entries indicate that the relative risk of pre-peak cases is significantly lower for the age-group in the row.

\begin{tabular}{|c|c|c|c|c|c|c|}
\hline \multicolumn{7}{|c|}{ Age group } \\
\hline$[1-5)$ & $9 / 100$ & & & & & \\
\hline$[5-10)$ & $100 / 100$ & $100 / 100$ & & & & \\
\hline$[10-15)$ & $100 / 100$ & $1 / 100$ & $100 / 100$ & & & \\
\hline$[15-20)$ & $100 / 100$ & $1 / 100$ & $4 / 100$ & 7/100 & & \\
\hline$[20-45)$ & $100 / 100$ & $1 / 100$ & $100 / 100$ & $100 / 100$ & $1 / 100$ & \\
\hline$>45$ & $3 / 100$ & $11 / 100$ & $3 / 100$ & $6 / 100$ & $2 / 100$ & $1 / 100$ \\
\hline & $<1$ & {$[1-5)$} & {$[5-10)$} & {$[10-15)$} & {$[15-20)$} & {$[20-45)$} \\
\hline
\end{tabular}

